\title{
Psychological capital, task autonomy and innovative work behaviour among public organisation employees
}

\section{Chiyem Lucky Nwanzu}

\author{
Department of Psychology, \\ Delta State University, \\ Abraka. Nigeria \\ Email: nwanzulc@delsu.edu.ng \\ Email: nwanzuchiyem@gmail.com
}

\section{Sunday Samson Babalola*}

\author{
School of Management Sciences, \\ University of Venda, \\ University Road, Thohoyandou 0950, South Africa \\ Email: sunday.babalola@univen.ac.za \\ *Corresponding author
}

\begin{abstract}
A fierce competitive workplace climate compels employees to work under pressure, hence, to perform requires individual's innovate positive psychological resources. This paper sets out to examine the predictive relationship between psychological capital (PsyCap) and innovative work behaviour (IWB) among public employees, and the role of task autonomy in the relationship. A cross-sectional survey design was conducted, and 125 employees from public hospitals participated in the study. The study used a questionnaire focussing on measures of PsyCap, IWB, task autonomy as well as questions on demographic characteristics. The research hypothesis was tested using regression analysis. Multiple regression analysis showed self-efficacy $(\beta=0.25, \mathrm{p}<0.01)$ and optimism $(\beta=0.14, \mathrm{p}>0.05)$ positively and significantly predict IWB. The investigation implicitly tested and confirmed the applicability of certain elements in Bandura's social cognitive theory and Vroom's expectancy theory from the examination of PsyCap - IWB link.
\end{abstract}

Keywords: psychological capital; PsyCap; innovative work behaviour; IWB; task autonomy; cognitive theory; expectancy theory.

Reference to this paper should be made as follows: Nwanzu, C.L. and Babalola, S.S. (2019) 'Psychological capital, task autonomy and innovative work behaviour among public organisation employees', Int. J. Work Organisation and Emotion, Vol. 10, No. 4, pp.322-338.

Biographical notes: Chiyem Lucky Nwanzu is a $\mathrm{PhD}$ holder in Industrial/Organizational Psychology and a lecturer in the Department of Psychology, Delta State University, Abraka, Nigeria. His research interests are workplace attitude and behaviour, organisation sustainability and change management. 
Sunday Samson Babalola is a Professor in the Department of Human Resource Management and Labour Relations, School of Management Sciences, University of Venda. $\mathrm{He}$ is a South African Rated Scientist. His research interests focus on, work ethics, entrepreneurship, workplace attitude, human capital management and organisational behaviour.

This paper is a revised and expanded version of a paper entitled 'Psychological capital, task autonomy and innovative work behaviour among health workers' presented at APS 13th Industrial and Organisational Psychology Conference, Adelaide, Australia, 11-13 July 2019.

\section{Introduction}

Positive psychology calls for adequate scientific interest in generations and accumulation of knowledge on human positive attributes for the benefit of all. In organisational studies, positive organisational behaviour (POB), as an emerging field of study, is attracting appreciable scholastic energy in terms of theory building and production of empirical studies. Luthans et al. (2007) conceptualised POB as the study and application of positively oriented human resource strengths and psychological capacities that can be measured, developed, and effectively managed for performance improvement in the workplace. The stringent requirements expressed in this definition, indicate that POB, is a narrow field. This remark has its basis in a number of psychological capabilities that do not yet have valid measures and reliability. While some psychological capabilities, such as, hope, self-efficacy, optimism and resilience, have been rigorously identified as meeting the criteria for inclusion in POB, a few others, like wisdom, humour and courage are noted to have the potentials for inclusion. Luthans et al. (2007) grouped these four variables that met the POB criteria as 'psychological capital' (PsyCap). This is defined as a positive mental developmental state of an individual that is characterised by hope, efficacy, and optimism.

PsyCap is the individual's positive psychological resources which are rooted in positive psychological movement (Donaldson and Ko, 2010). The movement has gained importance as positive psychology highlights the strengths, virtues, excellence, resilience and optimal functioning of an individual (Donaldson and Ko, 2010). PsyCap in work setting, thus, has been proposed as giving a competitive edge as it contributes to some desirable organisational outcomes. PsyCap - performance proposal has attracted some empirical work and confirmation. For instance, PsyCap predicts supervisors-rated performance (Luthans et al., 2005), innovation (Ziyae et al., 2015), knowledge sharing (Sharafi et al., 2014), and organisation learning (Mahar et al., 2017). Extensive research efforts have been made to decipher the value of PsyCap in work setting, however, this study is necessitated by some gaps in the existing literature. First, innovative work behaviour (IWB) is urgently needed as never before for organisations to survive in their very competitive and turbulent environments. There are, therefore, very practical reasons to understand the process, including the antecedents of IWB. Considering that individual dispositional traits have much implication for behaviour and that PsyCap has been implicated in a number of desirable organisational behaviour, understanding the relationship between PsyCap and IWB becomes imperative. Studies on PsyCap and IWB, 
however, are conspicuously deficient and there have been calls for more studies (Ratinaningsih et al., 2016; Sameer and Ohly, 2017). Second, with the few existing studies on PsyCap and IWB, there is limited assessment of their direct relationship and any intervening factors. This is one of the reasons Luthans et al. (2005) called for studies on mediating and moderating variables with regards to PsyCap issues. The call has since been attracting attention; therefore, this study sets out to add to the empirical studies on the relationship between PsyCap and IWB, especially, within a workplace environment where such research is lacking. Testing the relationship between PsyCap and IWB in the present research's location is necessary as culture and environment have implications on how research instruments are interpreted and the nature of relationships between variables. This study is also of value as it initiates the generation of knowledge on the mediating role of tasks autonomy in the relationship between PsyCap and IWB among public-organisation employees. A body of knowledge in this regard would be of value when there is need to manipulate PsyCap for IWB. Finally, this study offers the social cognitive and expectancy theories as theoretical explanations for the positive relationship between PsyCap and IWB. This investigation, thus, draws attention to the relevance of these theories in the relationship and by extension, tests and offers empirical confirmation to aspects of the theories that are relevant to PsyCap and IWB.

\section{Theoretical framework and research hypotheses}

\subsection{PsyCap-hope, optimism, self-efficacy and resilience}

Hope, from positive psychology perspective refers to a positive motivational state that result from the thinking that one has successfully set out appropriate goals and has identified possible and alternative ways to achieve these goals. The process of hope begins with identification of meaningful and desired future goals; it requires an individual to sufficiently envisage behaviour that would link present actions to future outcomes and to possess sufficient will power to pursue the various behaviours that could lead to the goal (Bloem, 2016; Bloem et al., 2018). Optimism is traditionally conceptualised as a broad personality trait characterised by general optimistic expectations. It expresses expectations that more good things than bad will happen in the future (Scheier and Carver, 1985). From a positive psychology perspective, optimism expresses an individual style of explaining experiences and can be attributed to either external or internal factors (Seligman, 1998). This perspective proposes that optimists hold external attributions (for example, prevailing circumstances) responsible for negative experience, while pessimists hold internal attributions (for example, personal failure) responsible for negative experience.

Self-efficacy is a person's belief in his/her capability of performing a particular task, successfully, within a given context (Bandura, 1997). It has three dimensions magnitude (the level of task difficulty an individual believes he/she can attain); strength (how strong or weak the individual believes the magnitude is) and generality (the extent the individual believes the capability can be generalised across situations). Individual self-efficacy is a function of past performance, vicarious experience, verbal persuasion, and emotional cues (Lunenburg, 2011). Various researchers have identified certain characteristics of self-efficacy that have implications for IWB. For instance, high self-efficacy brings with it the feeling that one is capability of doing whatever one wants 
to do, welcomes and thrives on challenge, is self-motivated, can invest necessary effort to accomplish and will persevere in face of obstacles (Akhtar et al., 2013; Luthans et al., 2007). Resilience is the ability to make a successful comeback after being overwhelmed by problems, failures, crises or even after positive events (Luthans et al., 2007). Resilient people accept reality that would increase their ability to survive; they belief that life is meaningful even in terrible times and have the ability to cope and improvise (Morsy, 2015). PsyCap constituents share certain characteristics but also differ in some ways. Hope and optimism have future orientation; characteristic lacking in the other elements of PsyCap. Self-efficacy and resilience implicate ability, characteristics absent from the other elements of PsyCap. Compared to others, optimism is least in state-like characteristics, while hope is the highest.

\subsection{Innovative work behaviour}

IWB refers to employee's actions that have implication for generation, introduction and application of new ideas, processes, products or procedures that are beneficial to a unit or an organisation (De Spiegelaere et al., 2014). Such behaviour, therefore, includes both introduction of new products and novel administrative procedure; it could range from small improvements such as task handling to radical novel ideas that could impact products or processes across the entire organisation. An innovative employee would exhibit behaviour reflecting a search for better alternative ways of carrying out tasks, seeking for better technology and work methods, concern for improvement on existing standards, seeking for resources needed for creation of new ideas, among others (Axtell et al., 2000). IWB among employees is of much value as it has positive implication on competitive advantage for organisations however, although much needed by employers most organisations do not formally demand such behaviour from their employees. IWB in most organisations is left to the discretion of the employees, who incubate it largely among other work behaviour referred to as 'organisational citizenship behaviour'; these are individual behaviours that are discretionary, not directly or overtly recognised by the formal reward system and that in the aggregate enhances organisational performance (Organ, 1988).

Social cognitive theory (Bandura, 1986) and expectancy theory (Vroom, 1964) offered explanations for the proposed relationship between PsyCap and IWB. Social cognitive theory proposes that personal factors, situational factors and behaviour, influence one another, and that individuals are best understood from their conscious cognitive capability (Cervone and Williams, 1992). One aspect of the personal factor is cognition; which refers to the way we think. The theory partly posits that how we think has implication for what we do. Social cognitive theory is applied to the variables of this study as PsyCap asserts that cognition is largely resultant from social processes. This study proposes a possible link between personal factors, social cognitive theory, PsyCap and IWB. Expectancy theory (Vroom, 1964) holds that the efforts employees put in carrying out their duties are functions of expectation of job outcomes, the desirability of the job outcome and the energy and ability to achieve the outcome (Roeckelein, 1998). Like cognitive social theory, the expectancy theory also proposes interactions between cognition, environment and behaviour, however, the cognitive element of the expectancy theory that has significant implication for this study is perceived ability to achieve outcome. The various dimensions of PsyCap have the potentials to express ability, 
therefore, it is proposed in this study that the cognitive processes that underly hope, self-efficacy, optimism, and resilience will have implications for IWB. In other words, the degree to which these variables exist in an individual will relate with the degree of IWB that the individual will exhibit.

\subsection{Task autonomy}

Job design refers to the process of laying out job responsibilities and duties and describing how they are to be performed (DuBrin, 2006). Among the common components of the various models of job design is task autonomy. Task autonomy refers to how much freedom and independence an employee has to carry out his or her work assignment. Such freedom could be in work scheduling, decision-making and work methods (Hackman and Oldham, 1980). In other words, task autonomy is the latitude employees have to decide about how to approach a piece of work. The degree of freedom an employee has, in carrying out job task have is dependent on how an employee experiments with activities associated with the task. Experimentation in work schedule, decision-making and work methods are IWB themselves, and they could also result in generation of ideas which are crucial aspect of innovativeness. Similarly, job autonomy gives more responsibility and could be source of motivation for certain individuals therefore, whatever factors have predictive influence on innovative behaviour have task autonomy to contend with.

Social exchange theory offers explanation for the proposed moderating role of task autonomy in any relationship between PsyCap and IWB. Social exchange refers to transaction or relationship between two or more parties (for example, relationships between employees and their organisation) that involves unspecified future obligations through a reciprocal process of exchanging resources (for example, reciprocity as interdependent exchanges) for which some future repayment or return is expected for the positive contribution made (Kim and Park, 2017). A basic principle of social exchange theory is the norm of reciprocity that obligates individuals to respond positively to favourable treatment received from another entity (Blau, 1964). Employees are in social exchange relationships with their organisations and when employees believe that they are treated fairly in the relationship they are motivated to give more of themselves (affectively, cognitively and behaviourally) in support of their supervisor, group, or organisation (Trevino and Brown, 2005). According to the theory, when employers provide their employees with positive work experiences, such as tasks autonomy, the employees would be under an obligation to reciprocate by engaging in IWB. The role of social exchange in autonomy - innovative behaviour relationship has been demonstrated in a number of studies. For instance, organisational procedural justice (Kim and Park, 2017), and workplace learning - job training and in-work learning opportunities (De Spiegelaere et al., 2014) have been found to be positively related to IWB.

\subsection{PsyCap, IWB and tasks autonomy}

Empirical work on the relationship between PsyCap, IWB and tasks autonomy is growing and with promising result for management practice. Investigation of relationship between 
PsyCap and innovative behaviour of employees by Hassan (2012) indicted that PsyCap significantly explained the variance in employee's innovative behaviour. Sameer and Ohly (2017) examined PsyCap and innovative behaviour among professionals from a variety of job types, in different companies; results showed a positive relationship between the variables. Similarly, Ziyae et al. (2015) observed that PsyCap as composite factors has significant effect on innovation, just as resilience enhanced innovation technology, while self-efficacy, hope and optimism did not. Dong et al. (2016) reported that composite PsyCap and its dimensions positively predict IWB, while knowledge sharing plays intermediary role between PsyCap and IWB.

Sweetman et al. (2011) reported that PsyCap and its components predicted creative performance while composite PsyCap predicted creative performance over and above each of the four components. Gupta and Singh (2014) found that PsyCap positively relates with IWB and fully mediates the relationship between leadership and creativity. The study of Zubair and Kamal (2017) also found that PsyCap and perceived authentic leadership were strong predictors of creative work behaviour while PsyCap mediated the relationship between perceived authentic leadership and creativeness. Rego (2009) also predicted the three dimensions (novel ideas, creative ideas and ideas championing) of creativity, in the same vein, the mediating 'way power' dimension of hope predicted the ideas championing dimension. Maymand et al. (2016) observed that while emotional intelligence, self-efficacy, optimism, subjective well-being and resilience positively and significantly impacted innovation, hope did not. Hsu et al. (2012) studied creative selfefficacy, optimism and innovative behaviour with 120 female employees and found that while creative self-efficacy had direct effect on innovative behaviour at work, optimism had no direct effect but mediated the effect of creative self-efficacy on employees' innovative behaviour. Federic (2013) observed a positive relation between principals' self-efficacy and perceived job autonomy. Saragih (2011) reported that self-efficacy partially mediated the relationship between job autonomy, job satisfaction and job performance. Binnewies and Gromer (2012) observed that job control predicted idea generation; support from co-workers and the supervisor predicted idea promotion, while co-worker and supervisor support predicted idea implementation. Sazandrishvili (2009) observed that job autonomy had positive effect on innovative behaviour while Tamunosiki-Amadi and Dede (2015) reported that self-determination had a significant positive relationship with idea generation and idea development, but weak significant relationship with idea implementation. Mee (2010) observed that job autonomy, proactive personality and openness to experience were positively related to IWB. The thesis of this study is that the degree of PsyCap in an employee would reflect such employee's IWB, and that the effect of PsyCap on IWB would change along with the level of task autonomy an employee's experiences. The following hypotheses, therefore, were tested:

Hypothesis (H1) PsyCap of hope, self-efficacy, optimism and resilience will positively and significantly predict IWB among public organisation employees.

Hypothesis (H2) Task autonomy will moderate the relationship between PsyCap of hope, self-efficacy, optimism, resilience and IWB among public organisation employees. 


\section{Research methodology}

\subsection{Sample and procedure}

One hundred twenty five employees sampled from some public hospitals in Delta State, Nigeria provided the data for the analyses. It comprises of $55 \%$ males and, $45 \%$, females; $72 \%$ married, and $28 \%$ single. Their age mean was 39.48 years (SD, 9.2 years). All the participants have had formal education with the majority (70\%) holding a first degree, its equivalent and other higher certificates. This is a literate sample and it gives validity to the self-report measure adopted. The sample included both junior and senior staff; this inclusiveness was to enhance generalisation of the findings.

On approval from the appropriate authorities, questionnaires were administered with the assistance of some administrative staff of the sampled organisations. A total of 150 questionnaires were administered to the participants at their workplaces. After an interval of three weeks, 132 of completed questionnaires were received, however, after sorting out those not appropriately completed, 125 were found useable for data analysis. The return rate is satisfactory as it exceeded survey response rate levels and trends in organisational research (Baruch and Holton, 2008). The participant sample size of 125 was considered satisfactory as it is in congruent with Dewberry's (2004) recommendation that when an effect size is unknown, the sample size required for a medium effect size should be adopted. The sample size adopted in the present study, therefore, has above $90 \%$ power of detecting a significant association between each pair ( $\mathrm{p}<0.05$ level) of significance, if such an association exists.

\subsection{Research instrument}

The 24-item scale of Luthans et al. (2007) was used to measure PsyCap. The scale items were phrased to reflect work-setting measure of four types (hope, optimism self-efficacy and resilience) of capital, and each of the capital had six items each. This scale has been adopted in studies that represent many nations and cultures and has been translated into a few languages (Antunes et al., 2017). Cetin and Basim's (2012) confirmatory factor analysis on the scale yielded satisfactory results in terms of structure, reliability and validity. Janssen's (2000) 9-item scale was used to measure IWB. This scale has three dimensions - idea generation, idea promotion and idea implementation. Each of the dimensions has three items. Breaugh's (1985) 9-item scale was used to measure task autonomy. The scale has three dimensions that covered - work method autonomy, work scheduling autonomy, and work criteria autonomy. It is a widely adopted in studies on work autonomy and has received satisfactory psychometric reports (Saragih, 2011).

A five-point Likert method of summated rating scale (5-strongly agree, 4-agree, 3-undecided, 2-disagree, 1-strongly disagree) was adopted as it usually, generates enough variability in responses, hence, gives validity to statistical outputs (Stone, 1978). Wide-scale points also control the effects of central tendency. For all the scales, scores were computed by averaging each participant responses to the items. Cronbach's alpha reliability coefficient of $\alpha=0.70, \alpha=0.78$, and $\alpha=0.74$ were obtained from composite PsyCap, IWB and task autonomy data respectively. Data on components of PsyCap yielded the following coefficient alpha: hope $\alpha=0.73$; optimism $\alpha=0.77$; self-efficacy $\alpha=0.52$; and resilience $\alpha=0.62$. Data on components of IWB yielded the following coefficients: idea generation $\alpha=0.74$, idea promotion $\alpha=0.72$, and idea implementation 
$\alpha=0.82$. These statistics indicate that the scales except for self-efficacy were of good reliability, as an alpha of $\alpha=0.70$ or above are considered satisfactory (Howitt and Cramer, 2011).

\subsection{Research design and data analysis}

This study adopted cross-sectional design as data were collected from the sample at one point in time. Cross sectional design is appropriate for this study as a survey has the potential for results' generalisation; this study also used non-random sampling technique (convenience sampling) in selection of both the organisations and the participants. It is a convenience sample because the participants used were on basis of availability. The use of non-random sample is a common feature in organisation studies, particularly as sampling frames are often not available or extremely difficult to access.

Hypothesis 1 was tested with simple and standard multiple regression, while hypothesis 2 was tested with hierarchical multiple regression. While the simple regression analysis provided information on relationship between the predictor variable(s) and the criterion variable(s) without extraction the possible influence of other related variable(s) on the predictor variable(s), the multiple regression analysis provided information on the relationship between the predictor variable(s) and criterion variable(s) when the influence of other related variables on predictor variable(s) were extracted. Multiple regression analysis gives understanding of how much other related variable(s) influenced the predictor variable(s) in its relationship with the criterion variable(s). Regression generally is a parametric test and assumptions associated with their usage were taken into cognisance. For instance, the assumption of data independence was met as every participant's response was independent of each other. Collection of data at interval level of measurement was achieved with the use of five-point Likert scaling format. Durbin-Watson test statistics that fall between 2.08 and 2.38 indicate absence of autocorrelation. Similarly, results from variance inflation factors (VIFs) which were below 10 and tolerance statistics which were above 0.2 indicate absence of collinearity in the data sets (Field, 2013).

\section{Results}

Table 1 shows statistics on mean, standard deviation and correlation coefficients on the research variables. All the pairs of relationships were significant from $p<0.05$ to $\mathrm{p}<0.01$. The highest degree of relationship was between PsyCap and optimism, while the least was between task autonomy and idea promotion, at $.013 \mathrm{p}<0.05$. The degree of correlations between the variables was modest, indicating the absence of multicollinearity in the model.

Table 2 showed simple regression analysis of hope, self-efficacy, optimism and resilience on IWB. As indicated by the individual variable, regression values, the relationship between hope and IWB $(\mathrm{R}=0.19, \mathrm{p}<0.01)$, self-efficacy and IWB $(\mathrm{R}=0.39, \mathrm{p}<0.01)$, optimism and IWB $(\mathrm{R}=0.50, \mathrm{p}<0.01)$, resilience and IWB $(\mathrm{R}=$ $0.37, \mathrm{p}<0.01)$ were positive and statistically significant. Analysis of variance (ANOVA) test for hope was $\mathrm{F}(1 ; 124)=4.64, \mathrm{p}<0.05$; self-efficacy, $\mathrm{F}(1 ; 124)=23.06, \mathrm{p}<0.01$; optimism, $\mathrm{F}(1 ; 124)=40.89, \mathrm{p}<0.01$, and resilience $\mathrm{F}(1 ; 124)=19.70, \mathrm{p}<0.01$. The 
ANOVA tests indicated that the individual regression was statistically significant; that is, IWB can be predicted from PsyCap elements.

Table 1 Mean, standard deviation and correlation coefficient on research variables

\begin{tabular}{llccccccccccc}
\hline & & $\bar{x}$ & $S D$ & 1 & 2 & 3 & 4 & 5 & 6 & 7 & 8 & 9 \\
\hline 1 & PsyCap & 3.78 & .46 & & & & & & & & & \\
2 & Hope & 3.92 & .63 & $.65^{* *}$ & & & & & & & & \\
3 & Optimism & 3.73 & .60 & $.65^{* *}$ & $.31^{* *}$ & & & & & & & \\
4 & Resilience & 3.72 & .67 & $.53^{* *}$ & $.33^{* *}$ & $.39^{* *}$ & & & & & & \\
5 & Efficacy & 3.92 & .61 & $.62^{* *}$ & $.34^{* *}$ & $.28^{* *}$ & $.36^{* *}$ & & & & & \\
6 & TaskA & 3.25 & .72 & $.30^{* *}$ & .19 & $.31^{* *}$ & .14 & $.23^{* *}$ & & & & \\
7 & IWB & 3.81 & .50 & $.51^{* *}$ & $.50^{* *}$ & $.19^{* *}$ & $.37^{* *}$ & $.39^{* *}$ & .15 & & & \\
8 & IdeaG & 3.87 & .59 & $.36^{* *}$ & $.23^{* *}$ & $.39^{* *}$ & $.22^{* *}$ & $.20^{* *}$ & .17 & $.43^{* *}$ & & \\
9 & IdeaP & 3.72 & .58 & $.35^{* *}$ & .15 & $.30^{* *}$ & $.31^{* *}$ & $.18^{* *}$ & .13 & $.47^{* *}$ & $.42^{* *}$ & \\
10 & IdeaImp & 3.72 & .66 & $.39^{* *}$ & $.22^{* *}$ & $.34^{* *}$ & $.25^{* *}$ & $.24 * *$ & $.19^{* *}$ & $.50^{* *}$ & $.43^{* *}$ & $.60^{* *}$ \\
\hline
\end{tabular}

Notes: $* *<.05$ level (two-tailed); PsyCap = psychological capital;

efficacy $=$ self-efficacy; Task $A=$ task autonomy; IWB $=$ innovative work

behaviour; IdeaG = idea generation; IdeaP = idea promotion;

Idealmp $=$ idea implementation

Table 2 Simple regression analysis on hope, self-efficacy optimism and resilience on innovative work behaviour and idea generation, idea promotion and idea implementation

\begin{tabular}{cccccc}
\hline & & Hope & Self-efficacy & Optimism & Resilience \\
\hline IWB & $R$ & $.19^{* *}$ & $.39^{* *}$ & $.50^{* *}$ & $.37^{* *}$ \\
& $R^{2}$ & .03 & .15 & .25 & .13 \\
& $B$ & .15 & .32 & .42 & .27 \\
& $S E$ & .07 & .06 & .06 & .06 \\
IG & $R$ & $.23 * *$ & $.20^{* *}$ & $.30^{* *}$ & $.22^{* *}$ \\
& $R^{2}$ & .05 & .04 & .09 & .04 \\
& $B$ & .22 & .20 & .30 & .19 \\
& $S E$ & .08 & .08 & .08 & .07 \\
IP & $R$ & $.15 * *$ & $.18^{* *}$ & $.30^{* *}$ & $.31^{* *}$ \\
& $R^{2}$ & .02 & .03 & .09 & .09 \\
& $B$ & .14 & .17 & .29 & .27 \\
& $S E$ & .08 & .08 & .08 & .07 \\
II & $R$ & $.22 * *$ & $.24 * *$ & $.34 * *$ & $.25^{* *}$ \\
& $R^{2}$ & .04 & .05 & .12 & .06 \\
& $B$ & .23 & .26 & .38 & .20 \\
& $S E$ & .09 & .09 & .09 & .09 \\
& & $F(1 ; 124)=4.64$, & $F(1 ; 124)=23.06, \quad F(1 ; 124)=40.89, \quad F(1 ; 124)=19.70$, \\
& $p<0.05$ & $p<0.01$ & $p<0.01$ & $p<0.01$ \\
\hline
\end{tabular}

Notes: Innovative work behaviour (IWB), idea generation, (IG), idea promotion (IP), and idea implementation (II). ${ }^{* *}<.05$ level (two-tailed) 
The $\mathrm{R}^{2}$ indicated that hope accounted for 3\% variance, self-efficacy $15 \%$ variance, optimism $25 \%$ variance and resilience $13 \%$ variance in IWB. On the basis of Cohen's (1988) criterion, $\mathrm{R}^{2}$ of $0.03,0.15,0.25$ and 0.13 indicate small, medium, large and medium effect sizes, respectively. The small difference in adjusted $R^{2}$ of $0.02,0.24,0.13$ and 0.15 respectively indicates a good cross validation, that is, the models have the potential to be applied to other samples from similar population. The B-values indicate that for everyone there was a unit increase in hope, self-efficacy, optimism and resilience, while on IWB increases were by $0.15,0.32,0.42$ and 0.27 respectively.

The other part of Table 2 showed results from simple regression analyses on the relationship between the dimensions of predictors on the criterion variable. The results from the dimensional analysis are of the same patterns with that of composite analysis. The relationship between organisational PsyCap of hope, self-efficacy, optimism and resilience and the dimensions of IWB (idea generation, idea promotion and idea implementation) were positive and significant.

Table 3 Multiple regression analysis of hope, self-efficacy optimism and resilience on innovative work behaviour, idea generation, idea promotion and idea implementation

\begin{tabular}{|c|c|c|c|c|c|}
\hline & & Hope & Self-efficacy & Optimism & Resilience \\
\hline \multirow[t]{4}{*}{ IWB } & $B$ & -.05 & $.21 * *$ & $.32 * *$ & .11 \\
\hline & $S E$ & .06 & .06 & 07 & .06 \\
\hline & $\mathrm{B}$ & -.06 & .25 & .39 & .14 \\
\hline & $\mathrm{PC}$ & -.06 & .23 & .34 & .12 \\
\hline \multirow[t]{4}{*}{ IG } & $B$ & .10 & .07 & $.22 * *$ & .05 \\
\hline & $S E$ & .08 & .09 & .09 & .08 \\
\hline & $\mathrm{B}$ & .11 & .08 & .22 & .06 \\
\hline & $\mathrm{PC}$ & .10 & .07 & .22 & .05 \\
\hline \multirow[t]{4}{*}{ IP } & $B$ & -.001 & .04 & $.18^{* *}$ & $.18^{* *}$ \\
\hline & $S E$ & .08 & .09 & .09 & .09 \\
\hline & $\mathrm{B}$ & -.001 & .05 & .19 & .20 \\
\hline & $\mathrm{PC}$ & -.001 & .04 & .17 & .16 \\
\hline \multirow[t]{5}{*}{ II } & $B$ & .08 & .12 & $.28 * *$ & .06 \\
\hline & $S E$ & .10 & .10 & .10 & .10 \\
\hline & $\mathrm{B}$ & .08 & .11 & .26 & .06 \\
\hline & $\mathrm{PC}$ & .07 & .10 & .22 & .05 \\
\hline & & $\begin{array}{c}F=15.36, R=.58, \\
R^{2}=.33, \\
\text { Adj. } R^{2}=.31 \\
\mathrm{p}<.05\end{array}$ & $\begin{array}{c}F=4.39, R=.35, \\
R^{2}=.12, \\
\text { Adj. } R^{2}=.09 \\
\mathrm{p}<.05\end{array}$ & $\begin{array}{c}F=4.51, R=.36 \\
R^{2}=.13 \\
\text { Adj } R^{2}=.10 \\
\mathrm{p}<.05\end{array}$ & $\begin{array}{c}F=5.27, R=.39, \\
R^{2}=.15, \\
\text { Adj } R^{2}=.12, \\
\mathrm{p}<.05\end{array}$ \\
\hline
\end{tabular}

Notes: Innovative work behaviour (IWB), idea generation, (IG), idea promotion (IP), and idea implementation (II). PC - Part Correlation. ${ }^{* *<}<.05$ level (two-tailed)

Multiple regression analysis in Table 3 showed IWB predicted from hope, self-efficacy, optimism and resilience. Self-efficacy and optimism positively and significantly predict IWB, while hope and resilience did not. Specifically, hope $(\beta=-0.06, p>0.05)$; self-efficacy $\beta=0.25, p<0.01)$; optimism $(\beta=0.39, p<0.01)$; and 
resilience $(\beta=0.14, \mathrm{p}>0.05)$. The $\beta$ value showed that the largest influence and statistically significant predictors on IWB were optimism and self-efficacy. Part correlation revealed that hope accounts for less than $1 \%$, self-efficacy $5 \%$, optimism $11 \%$ and resilience $1 \%$ variance in IWB. Table 3, also showed results from standard multiple regression analyses on the relationship between the predictors and dimensions of the criterion variable. The results from the dimensional analysis revealed that while all the organisational PsyCap have positive relationship with the dimensions of IWB, only organisational PsyCap of optimism has positive and significant relationship with the dimensions.

Table 4 showed hierarchical regression on the moderating role of tasks autonomy in the relationship between composite PsyCap, its dimensions and IWB. Composite analysis (moderating role of task autonomy on the relationship between PsyCap and IWB) revealed absence of moderating effect. Model 1 (without interaction) showed no significant relationship $\mathrm{F}(1,123)=3.11, \mathrm{p}>0.05$. Model 2 (with the interaction term) also revealed no significant relationship $\mathrm{F}(1,122)=0.17, \mathrm{p}>0.05$. The proportion of variance explained by the moderator or interaction effect $\left(\mathrm{R}^{2}\right.$ Change) was 0.001 . Compared to model 1 , model 2 did not significantly account for more variance, $\mathrm{R}^{2}$ change $=0.001, \mathrm{p}>0.05$. This indicates that task autonomy was not a potential moderator of the relationship between PsyCap and IWB.

Table 4 Hierarchical regression on moderating role of tasks autonomy in the relationship between psychological capital, its dimensions and innovative work behaviour

\begin{tabular}{|c|c|c|c|c|c|c|c|c|c|}
\hline & \multirow{2}{*}{ Model } & \multirow{2}{*}{$R$} & \multirow{2}{*}{$R^{2}-$} & \multirow{2}{*}{$\operatorname{Adj} R^{2}$} & \multicolumn{5}{|c|}{ Change statistics } \\
\hline & & & & & $R^{2}$ change & F change & $d f 1$ & $d f 2$ & Sig. $F$ \\
\hline PsyCap & 1 & .15 & .02 & .01 & .02 & 3.11 & 2 & 123 & $>.05$ \\
\hline PsyCap x TA & 2 & .16 & .02 & .01 & .001 & .17 & 1 & 122 & $>.05$ \\
\hline Hope & 1 & .22 & .05 & .03 & .05 & 3.28 & 2 & 122 & $<.05$ \\
\hline Hope x TA & 2 & .22 & .05 & .02 & .001 & .18 & 1 & 121 & $>.05$ \\
\hline Self-efficacy & 1 & .40 & .16 & .14 & .16 & 11.81 & 2 & 122 & $<.01$ \\
\hline Self-efficacy x TA & 2 & .41 & .17 & .14 & .007 & 1.08 & 1 & 121 & $>.05$ \\
\hline Optimism & 1 & .50 & .25 & .23 & .25 & 20.28 & 2 & 122 & $<.01$ \\
\hline Optimism x TA & 2 & .50 & .25 & .23 & .001 & .007 & 1 & 121 & $>.05$ \\
\hline Resilience & 1 & .38 & .14 & .13 & .14 & 10.65 & 2 & 122 & $<.01$ \\
\hline Resilience x TA & 2 & .39 & .15 & .13 & .004 & .50 & 1 & 121 & $>.05$ \\
\hline
\end{tabular}

Note: Organisational psychological capital (PsyCap), task autonomy (TA)

Dimensional analysis of moderating effect of task autonomy in the relationship between hope, self-efficacy, optimism, resilience and IWB revealed that while, model 1 (without interaction) for the dimensions showed significant relationship, model 2 (with the interaction term) revealed no significant relationship. The proportion of variance explained by the moderator or interaction effect as indicated by $\mathrm{R}^{2}$ Change for each of the dimensions was not significant. This indicates that task autonomy is not a potential moderator in the relationship between PsyCap of hope, self-efficacy, optimism, resilience and IWB. The $\mathrm{R}^{2}$ change for model 2 (interaction) for both composite and dimensional analyses indicate that the variance PsyCap (and the dimensions) separately accounted for in IWB by the interaction was less than $1 \%$. As the moderation effect was not significant, 
there was no additional analysis to understand the nature of the moderation (Howitt and Cramer, 2011).

\section{Discussion and conclusions}

This study examined the predictive relationship PsyCap has with IWB and the moderator role of task autonomy in the relationship. Descriptive statistics revealed moderate level of hope, self-efficacy, optimism, resilience, IWB and task autonomy among the participants. Data analysis revealed that the four dimensions of PsyCap relate positively and significantly with each other. This points to the possibility that effort to enhance any could lead to enhancing others. The relatedness of the dimensions is also expressed in their mean scores that were within the same range as shown in Table 1.

On hypothesis 1, which state that PsyCap and its dimensions have positive and significant predictive relationships with IWB was supported. PsyCap as a composite and in its dimensions have positive and significant predictive relationship with IWB. This result is congruent with the extant literature. For instance, Dong et al. (2016) reported that composite PsyCap and the dimensions positively predict IWB. Sweetman et al. (2011) reported that PsyCap and its components predicted creative performance and that composite PsyCap predicted creative performance over and above each of the four components. Gupta and Singh (2014) found that PsyCap positively relates with IWB. The composite analysis showed that PsyCap accounted for a large percentage of variance in IWB. On the basis that a number of variables are likely to impact IWB, the variance of PsyCap accounted for in IWB could be adjudged substantial and of practical value. The dimensional analysis which was achieved through standard multiple regression revealed that among the four dimensions of organisational PsyCap optimism accounted for the highest variance and resilience accounted for the lowest in IWB.

The hypothesis on whether task autonomy moderates the predictive relationship between organisational PsyCap, its dimensions and IWB was not supported. The observations were contrary to expectation. Two plausible explanations for the unexpected result could be offered. First, the sample size adopted in the study has a $90 \%$ power $(p<0.05)$ when the population effect size is medium (Dewberry, 2004). The effect sizes observed for model 2 (interaction) for the composite and dimensional analysis were all below small effect. The statistics indicate that the sample size adopted is much unlikely to seem 'significance' even when it does exist. As Dewberry (2004) reported, a sample size of 640 is required to detect a significant prediction when it does exist for a study with five predictors and a small effect size. Second, the variance moderator account for in any analysis is typically small as it is the variance which is left over after considering the variances of the independent variables (Jex, 2002).

Some conclusions could be reached from the findings of this study. From the results of both simple and multiple regression analyses it could be concluded that organisational PsyCap of hope, self-efficacy, optimism and resilience are important predictors of IWB. Second, from the multiple regression analysis it could be concluded that among the four dimensions of PsyCap, optimism has the greatest unique contribution in predicting IWB, while resilience has the lowest contribution, which is also not significant. Third, the relationships among the four dimensions of PsyCap were moderate, positive and significant. It could be concluded that effort to enhance any could have positive 
implication for the others. Similarly, the relationships among the three dimensions of IWB were moderate, positive and significant. Finally, considering the need for IWB, the observed effect sizes are meaningful and important. It has been abundantly noted in the literature (Ellis, 2010), that the practical utility of effect size is dependent on context, rather than its magnitude.

\subsection{Theoretical implications}

This study has some implications for theory development and confirmation. First, literature on the link between PsyCap and IWB is dominated by findings indicating positive relationship (Dong et al., 2016; Sweetman et al., 2011). The present study confirmed the trend in the findings as it revealed that PsyCap and its four constituents positively and significantly predict IWB. Consequently, this study contributes to a pattern of findings that could lead to middle range theorising on PsyCap - IWB relationship. Second, the findings that PsyCap positively predict IWB offers support and confirmation to certain aspects of Bandura's (1986) social cognitive theory. According to the theory, the way and manner individual thinks has much implication for their behaviour. The various constituents of PsyCap (hope, self-efficacy, optimistic and resilience) represent personal characteristics with potential for innovation. Third and related to the above, this study also provides confirmation for Vroom's expectancy theory which also proposes interactions between cognition, environment and behaviour, and their perceived ability to achieve outcome. Evidently, the various dimensions of PsyCap are embedded with the potentials to result in ability for desired outcomes. Forth, although it was hypothesised in this study, is that task autonomy will moderate the relationship between PsyCap of hope, self-efficacy, optimism, resilience and IWB, but that was not confirmed by the data. This finding reaffirms the domineering influence of personality on behaviour and disconfirmed situational strength theory which posits that an individual can act in certain manner despite his/her personality. The finding shows that neither a strong nor weak situation as represented in low and high autonomy respectively will influence how PsyCap relates with IWB. The current finding collaborates with that of Meyer et al. (2014) who observed a reversed effect of situation strength as a moderator in a relationship between consciousness, agreeableness personality traits and counterproductive work behaviour, and Chevalking (2019) who observed that clear (low situation strength) and non-ambiguous (high situation strength) signals did not lead individuals to show intention to use healthcare information technology. Some researchers, such as Judge and Zapata (2015) confirmed the situation strength theory prediction however the present study and others that observed disconfirming results for situation strength theory indicate the need for more empirical studies on situation strength theory.

\subsection{Recommendation for practice}

IWB is of much value in organisations, as it is needed for competitive advantage. Results (including those from this study) indicated that PsyCap positively and significantly predicted IWB. It is therefore recommended that human resource management practices make use of employees with appropriate levels of PsyCap in the organisation. This is feasible through recruitment, selection and training activities. Numerous approaches on how PsyCap can be enhanced exist in the literature (Luthens et al., 2006; Steenveld, 
2017). The various types of PsyCap differ in the size of variance explained in IWB among public organisations' employees. Both simple and multiple regression analyses show that optimism contributes greatest in the prediction of IWB, therefore efforts to enhance PsyCap among public organisation employees should give high attention to optimism dimension.

\subsection{Limitations and future research}

This study has some limitations which point to directions for future studies. First, data were collected with self-report measure, a method of data collection associated with common method bias. Common method bias refers to deviation in observed relationship from true relationship that is caused by the similarity in methods used to obtain the data. Common method bias is well adjudged to have negative impact on the validity of research findings as it inflates relationship between variables. Social desirability bias, halo effect and same source variance are various sources of common method bias. Future studies should include social desirability scales in attempts to identify and removed respondents with social desirability bias tendency (Dodd-McCue, 2010). Same source variance could be addressed by at least having the independent and dependent variables in separate questionnaires (Podsakoff et al., 2012). The halo effect could be addressed with appropriate wording of questionnaire items. In sum, future studies should adopt data triangulation. The designs of this study being cross-sectional which does not have the capacity to identify cause-effect relationship, therefore, future studies should explore quasi-experiment and longitudinal study that would enable causal interpretation. Task autonomy was not identified as a moderator in the tested relationships. More studies should be conducted on that relationship, other moderators and mediators should be theoretically and empirical proposed and examined in future studies. The adopted sample size can only see significance if the population effect size is medium. Further studies should use sample size that can detect significance if it does exist even when the population effect size is small.

\section{References}

Akhtar, S., Ghayas, S. and Adil, A. (2013) 'Self-efficacy and optimism as predictors of organisational commitment among bank employees', International Journal of Research Studies in Psychology, Vol. 2, No. 2, pp.33-42.

Antunes, A., Caetano, A. and Cunha, M.P. (2017) 'Reliability and construct validity of the Portuguese version of the psychological capital questionnaire', Psychological Reports, Vol. 120, No. 3, pp.520-536.

Axtell, C.M., Holman, D.J., Ushworth, K.L., Wall, T.D., Waterson, P.E. and Harrington, E. (2000) 'Shopfloor innovation: facilitating the suggestion and implementation of ideas', Journal of Occupational Organisational Psychology, Vol. 73, No. 3, pp.265-285.

Bandura, A. (1986) Social Foundations of Thought and Action, Prentice Hall, Englewoods, Cliffs.

Bandura, A. (1997) Self-efficacy: The Exercise of Control, W.H. Freeman, New York.

Baruch, Y. and Holton, C.B. (2008) 'Survey response rate levels and trends in organisational research', Human Relations, Vol. 61, No. 8, pp.1139-1160.

Binnewies, C. and Gromer, M. (2012) 'Creativity and innovation at work: the role of work characteristics and personal initiative', Psicothema, Vol. 24, No. 1, pp.100-105.

Blau P. (1964) Exchange and Power in Social Life, Wiley, New York. 
Bloem, J. (2016) 'Review of economic rules: the rights and wrongs of the dismal science by Dani Rodrik', Faith and Economics, Fall, Vol. 68, pp.121-126.

Bloem, J.R., Boughton, D., Htoo, K., Hein, A. and Payongayong, E. (2018) 'Measuring hope: a quantitative approach with validation in rural Myanmar', The Journal of Development Studies, Vol. 54, No. 11, pp.2078-2094.

Breaugh, J.A. (1985) 'The measurement of work autonomy', Human Relations, Vol. 38, No. 6, pp.551-570.

Cervone, D. and Williams, S.L. (1992) 'Social cognitive theory and personality', in Caprara, G.V. and Van Heck, G.L. (Eds.): Modern Personality Psychology: Critical Reviews and New Directions, pp.200-252, Harvester Wheatsheaf, New York.

Cetin, F. and Basim, H.N. (2012) 'Organisational psychological capital: a scale adaptation study', Amme İdaresi Dergisi, Vol. 45, No. 1, pp.121-137.

Chevalking, L. (2019) The Influence of Situation Strength on Adoption of Healthcare Information Technology, University of Twente, Australian School of Business, UNSW, Sydney.

Cohen, J. (1988) Statistical Power Analysis for the Behavioural Sciences, 2nd ed., Lawrence Erlbaum: Hillsdale, NJ.

De Spiegelaere, S., Van Gyes, G. and Van Hootegem, G. (2014) 'Innovatief werkgedrag als concept: definiëring en oriëntering', Gedrag en Organisatie, Vol. 27, No. 2, pp.139-156.

Dewberry, C. (2004) Statistical Methods for Organisational Research: theory and Practice, Routledge, New York.

Dodd-McCue, D. (2010) 'Self-report response bias: learning how to live with its diagnosis in Chaplaincy research', E Journal of the Association of Professional Chaplains, Vol. 26, No. 1, pp.1-7.

Donaldson, S.I. and Ko, I. (2010) 'Positive organizational psychology, behaviour, and scholarship: a review of the emerging literature and evidence base', The Journal of Positive Psychology, Vol. 5, No. 3, pp.177-191.

Dong, B., Wang, X-F. and Ding, Y. (2016) 'Research on the relationship between psychological capital, knowledge sharing and innovation behaviour of logistics enterprises employees', Advances in Social Science, Education and Humanities Research, Vol. 8, No. 7, pp.1-7.

DuBrin, J.A. (2006) Essentials of Management, 9th ed., South-Western, Australia.

Ellis, D.P. (2010) The Essential Guide to Effect Sizes: statistical Power, Meta-analysis, and the Interpretation of Research Results, Cambridge University Press, Cambridge.

Federic, A.R. (2013) 'Principals' self-efficacy: relations with job autonomy, job satisfaction, and contextual constraints', European Journal of Psychology of Education, Vol. 28, No. 1, pp.73-86.

Field, A. (2013) Discovering Statistics using IBM SPSS Statistics, 4th ed., Sage, Los Angeles.

Gupta, V. and Singh, S. (2014) 'Psychological capital as a mediator of the relationship between leadership and creative performance behaviours: empirical evidence from the Indian R\&D sector', The International Journal of Human Resource Management, Vol. 25, No. 10, pp.1373-1394.

Hackman, J.R. and Oldham, G.R. (1980) Work Redesign, Addison-Wesley, Reading, MA.

Hassan, J. (2012) 'Psychological capital and innovative behaviour: an empirical study on apparel fashion industry', Journal of Contemporary Management Research, Vol. 6, No. 1, pp.42-52.

Howitt, D. and Cramer, D. (2011) Introduction to SPSS Statistics in Psychology for Version 19 and Earlier, 5th ed., Pearson, New York.

Hsu, M.L.A., Hou, S-T. and Fan, H-L. (2012) 'Creative self-efficacy and innovative behaviour in a service setting: optimism as a moderator', Journal of Creative Behaviour, Vol. 45, No. 4, pp.258-272.

Janssen, O. (2000) 'Job demands, perceptions of effort-reward fairness and innovative work behaviour', Journal of Occupational and Organisational Psychology, Vol. 73, No. 3, pp.287-302. 
Jex, M.S. (2002) Organisational Psychology: A Scientist-Practitioner Approach, John Wiley and Son, Canada.

Judge, A.T. and Zapata, P.C. (2015) 'The person-situation debate revisited: effect of situation strength and trait activation on the validity of the big five personality traits in predicting job performance', Academy of Management Journal, Vol. 58, No. 4, pp.1149-1179.

Kim, W, and Park, J. (2017) 'Examining structural relationships between work engagement, organisational procedural justice, knowledge sharing, and innovative work behaviour for sustainable organisations', Sustainability, Vol. 9, No. 205, pp.1-6.

Lunenburg, C.F. (2011) 'Self-efficacy in the workplace: implications for motivation and performance', International Journal of Management, Business and Administration, Vol. 14, No. 1, pp.1-6.

Luthans, F., Avolio, B.J., Walumbwa, O.F. and Li, W. (2005) 'The psychological capital of Chinese workers: exploring the relationship with performance', Management and Organization Review, Vol. 1, No. 2, pp.249-271.

Luthans, F., Youssef, C.M. and Avolio, B.J. (2007) Psychological Capital: Developing the Human Competitive Edge, Oxford University Press, Oxford.

Luthens, F., Vogelgesang, R.V. and Lester, B.P. (2006) 'Developing psychological capital of resilience', Human Resource Development Review, Vol. 5, No. 1, pp.25-44.

Mahar, S.W., Basheer, S. and Maitlo, A.L. (2017) 'The influence of psychological capital on organisation learning in banking sector of Pakistan', Review of Integrative Business and Economics Research, Vol. 6, No. 4, pp.306-316.

Maymand, M., Moghadam, S.N., Farangi, A. and Rouholamin, M. (2016) 'Investigating the effect of positive organisation behaviour on innovation', International Business Management, Vol. 10, No. 11, pp.2282-2292.

Mee, S.L. (2010) Determinants of Innovative Work Behaviour among White-Collar Workers: A Study of the Manufacturing Firms within the Electrical and Electronic Sector of Penang, Unpublished Master of Business Administration Dissertation, Universiti Sains Malaysia.

Meyer, R.D., Dalal, R.S., José, I.J., Hermida, R., Chen, T.R., Vega, R.P. and Khare, V.P. (2014) 'Measuring job-related situational strength and assessing its interactive effects with personality on voluntary work behaviour', Journal of Management, Vol. 40, No. 4, pp.1010-1041.

Morsy, Y.M.S. (2015) Innovation, Creativity, and Positive Psychological Capital: examining the Relationships in Adolescents, Employees and Entrepreneurs, Unpublished Doctorate dissertation, Faculty of Economics and Management, University of Kassel. Germany.

Organ, W.D. (1988) Organisational Citizenship Behaviour: the Good Soldier Syndrome, Lexington Books, Lexington MA.

Podsakoff, P.M., MacKenzie, S.B. and Podsakoff, N.P. (2012) 'Sources of method bias in social science research and recommendations on how to control it', Annual Review of Psychology, Vol. 65, pp.539-569.

Ratinaningsih, Z.K., Prasetyo, R.A. and Prihat, U. (2016) 'Predicting innovative behaviour among employees in a manufacturing company: the role of psychological capital Indonesian', Psychological Journal, Vol. 31, No. 2, pp.84-90.

Rego, A. (2009) 'Are hopeful employees more creative? an empirical study', Creativity Research Journal, Vol. 21, Nos. 2-3, pp.223-231.

Roeckelein, E.J. (1998) Dictionary of Theories, Laws and Concepts in Psychology, Greenwood Press, London.

Sameer, Y.M. and Ohly, S. (2017) Innovative Behaviour of Employees: A Model of Antecedents and Consequences, a Deeper Look at Psychological and Organisational Factors, Faculty of Management Technology, German University in Cairo, Egypt, Working Papers Series No. 45.

Saragih, S. (2011) 'The effects of job autonomy on work outcomes: self-efficacy as an intervening variable', International Research Journal of Business Studies, Vol. 4, No. 3, pp.203-215. 
Sazandrishvili, N. (2009) Contextual and Personal Antecedents of Innovative Behaviour: Mediation Effect of Learning Goal Orientation on the Relationship between Job Autonomy and Innovative Behaviour, Unpublished dissertation, University of Twente.

Scheier, M.F. and Carver, C.S. (1985) 'Optimism, coping and health: assessment and implications of generalized outcome expectancies', Health Psychology, Vol. 4, No. 3, pp.219-247.

Seligman, M. (1998) Learned Optimism, Pocket Books, New York.

Sharafi, A., Mahabadi, M.N., Forouhar, M., Naeini, S.G. and Riahi, S. (2014) 'Explain the role of psychological capital in knowledge sharing of an organisation', International Journal of Management Academy, Vol. 2, No. 1, pp.51-57.

Steenveld, M. (2017) Psychological Capital: Training the 4 Positive Capabilities [online] $\mathrm{https} /$ positiveprogram.com./psycap/ (accessed 3 October 2018).

Stone, E. (1978) Research Methods in Organisational Behaviour, Scott, Foresman, Glenview, IL.

Sweetman, D., Luthans, F., Avey, J.B. and Luthans, B.C. (2011) 'Relationship between positive psychological capital and creative performance', Canadian Journal of Administrative Sciences/Revue Canadienne des Sciences de l'Administration, Vol. 28, No. 1, pp.4-13.

Tamunosiki-Amad, O.J. and Dede, T. (2015) 'Self-determination and employee innovative behaviour in the Nigerian telecommunication industry', European Journal of Business and Management, Vol. 7, No. 35, pp.97-105.

Trevino, L.K. and Brown, M.E. (2005) 'The role of leaders in influencing unethical behaviour in the workplace', in Kidwel, R.E. and Martin, C.L. (Eds.), Managing Organisational Deviance, Sage, California, USA.

Vroom, V. (1964) Work and Motivation, Wiley, New York.

Ziyae, B., Mobaraki, H.M. and Saeediyoun, M. (2015) 'The effect of psychological capital on innovation in information technology', Journal of Global Entrepreneurship Research, Vol. 5, No. 8, pp.1-12.

Zubair, A. and Kamal, A. (2017) 'Perceived authentic leadership, psychological capital, and creative work behaviour in bank employees', Pakistan Journal of Psychological Research, Vol. 32, No. 1, pp.35-53. 